\title{
Erasure of memory in paste by irradiation of ultrasonic waves
}

\author{
Akio Nakahara ${ }^{1, *}$, Ryota Yoneyama $^{1}$, Maruto Ito $^{1}$, Yousuke Matsuo ${ }^{1}$ and So Kitsunezaki ${ }^{2}$ \\ ${ }^{1}$ Laboratory of Physics, College of Science and Technology, Nihon University, Funabashi 274-8501, Japan \\ ${ }^{2}$ Research Group of Physics, Division of Natural Sciences, Faculty of Nara Women's University, Nara 630-8506, Japan
}

\begin{abstract}
Densely packed colloidal suspension, called paste, remembers the direction of applied forces, such as vibration and flow, and these memories kept in paste can be visualized as morphology of desiccation crack patterns. For example, when the paste remembers the direction of vibration, all primary cracks propagate in the direction perpendicular to the direction of initial vibration. On the other hand, when the paste remembers the direction of flow, all primary cracks propagate along the direction of initial flow. These results indicate that external forces imprint easy-breakable direction into paste as memories. Therefore, by controlling memories in paste, we can tune to produce various types of crack patterns, such as cellular, radial, lamellar, ring, spiral and lattice structures. Recently we have found that memories in paste can be erased by the irradiation of ultrasonic waves to paste as we obtain only isotropic and cellular crack patterns without any anisotropy related to memory effect. This method can be applied to increase the breaking strength of dried paste by homogenizing microstructure in paste.
\end{abstract}

\section{Introduction}

The morphology of crack patterns contains variety of information, such as a position of crack nucleation and the direction of crack propagation, but it is a difficult work to derive more useful information from them. Here we deal with crack patterns of dried paste. We study what kinds of information we can get from these crack patterns and try to control the morphology of crack patterns to prevent fatal crack formation.

When a densely packed colloidal suspension, called paste, is poured into the container and is dried at room temperature and at low humidity for few days, the paste shrinks as the water evaporates, and if the paste is stick to the bottom of the container, desiccation crack patterns emerge so as to release the stress induced by the shrinkage [1]. Figure 1 shows the typical example of desiccation crack pattern, such as the one of calcium carbonate paste dried in laboratory.

Usually the morphology of desiccation cracks is isotropic and cellular as is shown in Fig. 1. The final spacing between cracks is proportional to the thickness of the paste as long as the paste is not so thick [2].

On the other hand, when the thickness of the paste is large, a crack front propagates from the surface toward the inside along the drying direction, and, as for the case of starch paste, the columnar joint of hexagonal structure emerges, which looks similar to the columnar joint of cooled lava [1, 3-6]. In each case, materials shrink under drying or cooling, and the directions of drying or cooling gradient produce anisotropic crack structure along the gradient, such as columnar joints. The diameters of these columns are determined by the value of drying or cooling gradient. Therefore, the directions of these columns indicate in which direction the paste was dried and the lava was cooled.

Can we control the direction of crack propagation without any drying gradient? If we can, we can protect products from serious damages even if we cannot stop crack formation.

In this manuscript, we report that, even without any drying gradient, we can control and get regular and anisotropic desiccation crack patterns, such as radial, lamellar, ring and spirals. We have found that paste remembers the direction of external forces and the morphology of desiccation crack patterns is determined by the memory stored in paste. These results mean that, if we imprint or rewrite the memory in paste, we can order cracks to propagate along any directions we want [7]. Then we report our latest findings that memories in paste can be erased by the irradiation of ultrasonic waves. We will conclude with its further application to control the breaking strength of materials.

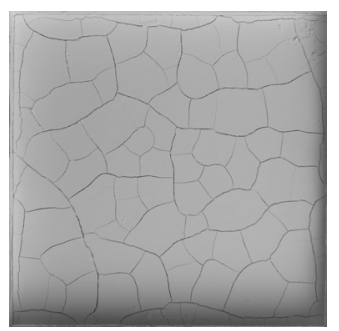

Fig. 1. Desiccation crack pattern of calcium carbonate paste. The size of the figure corresponds to $200 \mathrm{~mm} * 200 \mathrm{~mm}$.

Corresponding author: nakahara.akio@nihon-u.ac.jp 


\section{Memory effect of paste}

One day we performed drying experiments of calcium carbonate paste. The median diameter of colloidal particles is $2 \mu \mathrm{m} .3 \mathrm{~kg}$ of calcium carbonate powder is mixed with $1.5 \mathrm{~kg}$ of distilled water and the mixture is poured into the circular acrylic container with $500 \mathrm{~mm}$ in diameter. Since the paste is water-poor, we had to vibrate the circular container in the angular direction to spread the paste homogeneously throughout the container and to get a flat surface of the paste. The thickness of the paste is $13 \mathrm{~mm}$ after the vibration. Then, we stopped vibration, dried the paste at room temperature and at low humidity of $30 \%$. Two days later desiccation cracks appear, but surprisingly the pattern was not the isotropic and cellular one, the pattern was radial, as is shown in Fig. 2(a).

At first, we thought that we get radial crack pattern because we used a circular container. However, we noticed that we had vibrated the paste to spread it before drying, so we did similar drying experiment but this time we vibrated the paste horizontally in one direction. Then we get lamellar cracks as is shown in Fig. 2(b) and all primary cracks run in the direction perpendicular to the direction of the initial vibration.

Therefore, we realized that the water-poor paste with plasticity can remember the direction of the vibration applied to it, and the memory of the vibration can be visualized as morphology of desiccation crack patterns. When the paste remembers the direction of the vibration, all primary cracks run in the direction perpendicular to the direction of the initial vibration [7].

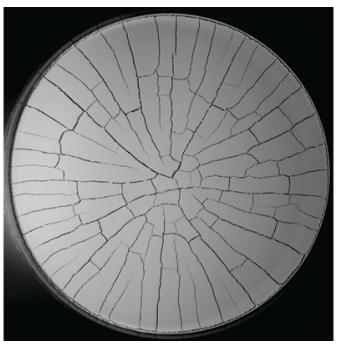

(a) Radial crack pattern

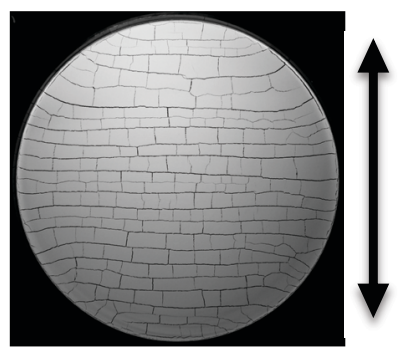

(b) Lamellar crack pattern
Fig. 2. Desiccation crack patterns of calcium carbonate paste. The diameter of each container is $500 \mathrm{~mm}$. (a) Radial crack pattern. (b) Lamellar crack pattern. The arrow indicates the direction of the initial vibration.

\subsection{Memory of vibration}

To reveal the condition when paste remembers the direction of the vibration, we performed systematic experiments by controlling two parameters, solid volume fraction of particles in paste and the strength of the initial vibration, and the result is shown in Fig. 3.

To prepare the paste, we mix calcium carbonate powder of $360 \mathrm{~g}$ with water and set the paste into a square acrylic container with each side $200 \mathrm{~mm}$. After the container is horizontally vibrated in one direction for 1 min., the paste is dried at room temperature and desiccation cracks appear in two days.
In Fig. 3, open circles in regions $\mathrm{A}$ and $\mathrm{C}$ represent isotropic and cellular cracks, while solid squares in region $\mathrm{B}$ represent lamellar cracks with the direction perpendicular to the direction of the initial vibration. The solid line denotes Yield stress line on which the shear stress induced by the initial vibration equals the yield stress of the paste. Here the yield stress of paste is an increasing function of the solid volume fraction in paste and is estimated by rheological measurements using Rheometer Physica MCR-301 (Anton Paar, Graz, Austria). We find that, in region B when the water-poor plastic paste is vibrated at strength just above the yield stress of the paste, the paste experiences and remembers the motion of vibration, and the memory of vibration stored in paste can be visualized as morphology of desiccation cracks [7-9]. This experimental result means that the plasticity of paste plays important role in memory effect. Theoretical approaches based on residual tension models are proposed to explain how the memory of vibration remains as a form of plastic deformation in paste $[1,10-12]$.

The boundary between regions $\mathrm{B}$ and $\mathrm{C}$, denoted by broken line, corresponds to hydrodynamic instability from the motion of vibration to flow. We find that, once calcium carbonate paste flows, it cannot remember any motions.

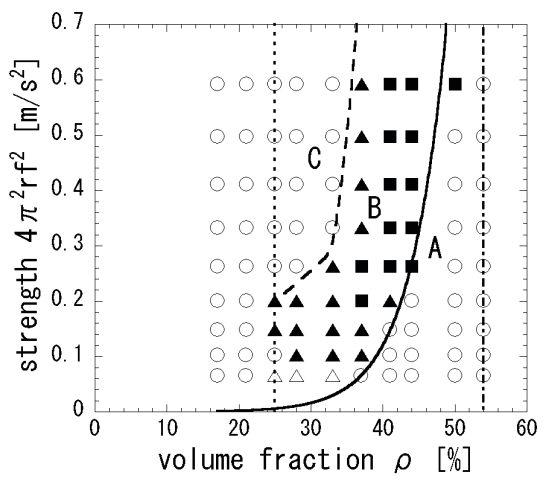

Fig. 3. Morphological phase diagram of desiccation crack patterns of calcium carbonate paste, as function of the solid volume fraction in paste and the strength of the initial vibration. Open circles represent isotropic and cellular cracks, solid squares represent lamellar cracks, the direction of which is perpendicular to the direction of the initial vibration (memory of vibration), and solid and open triangles represent combinations of cellular and lamellar cracks. The vertical dotted line denotes Liquid-Limit, the vertical dash-and-dotted line denotes Plastic-Limit, the solid line denotes Yield-stress line, and the broken line denotes the boundary between regions $\mathrm{B}$ and $\mathrm{C}$. Calcium carbonate paste remembers the direction of initial vibration when it was vibrated in region $\mathrm{B}$ [7].

\subsection{Memory of flow}

After the discovery of the memory effect of vibration, we had performed many drying experiments by using various type of paste, and found that some pastes remember not only the direction of vibration but also the direction of flow. When the paste remembers the flow direction, the primary desiccation cracks run in the direction parallel to the flow direction. Figure 4 show the spiral crack pattern produced by using the memory of 
spiral flow motion. To imprint the spiral motion into the paste, we had translated the container along a circuit with a radius of $15 \mathrm{~mm}$ clockwise.

To understand the mechanism how such pastes remember the flow direction, we performed systematic experiment to obtain a morphological phase diagram of desiccation cracks as functions of solid volume fraction and the strength of the initial vibration applied to the container. The experimental result using the paste of magnesium carbonate hydroxide is shown as Fig. 5.

Figure 5 shows that this paste has two type of memories. In region B when the water-poor paste is vibrated at strength just above the yield stress of the paste, the paste shows the same type of memory effect of vibration that the calcium carbonate paste has. On the other hand, in region $\mathrm{C}$ when the water-medium paste is fluidized by the vibration of the container, the paste remember the direction of the flow motion, and the primary desiccation cracks run in the direction parallel to the initial flow [13].

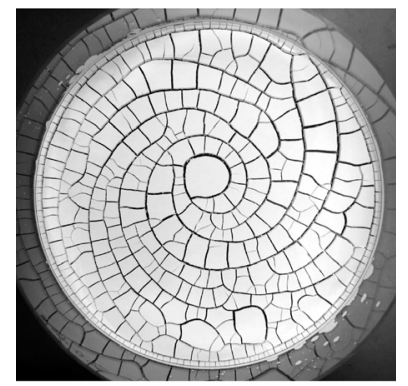

Fig. 4. Spiral crack pattern of paste of magnesium carbonate hydroxide. The diameter of the container is $500 \mathrm{~mm}$ [13].

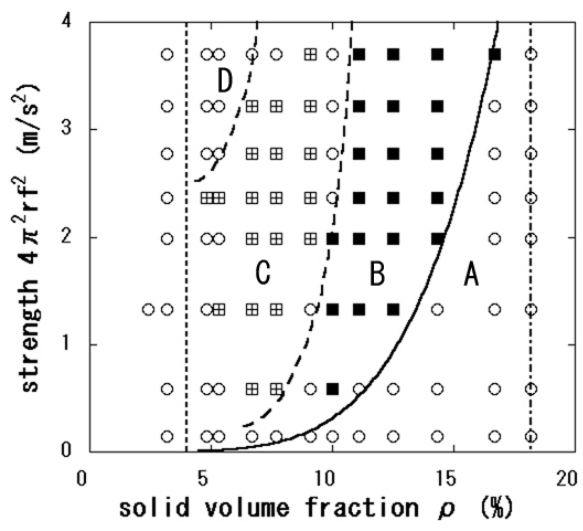

Fig. 5. Morphological phase diagram of the desiccation crack patterns of paste of magnesium carbonate hydroxide, as function of the solid volume fraction in paste and the strength of the initial vibration. Open circles represent isotropic and cellular cracks, solid squares represent lamellar cracks, the direction of which is perpendicular to the direction of the initial vibration (memory of vibration), and open squares with plus inside represent lamellar cracks, the direction of which is parallel to the direction of the initial flow. The vertical dotted line denotes Liquid-Limit, the vertical dash-and-dotted line denotes Plastic-Limit, the solid line denotes Yield-stress line, and broken lines denote the boundary between regions $\mathrm{B}$ and $\mathrm{C}$ and that between regions $\mathrm{C}$ and D. Paste of magnesium carbonate hydroxide can remember not only the direction of vibration but also the direction of the initial flow [13].
Note that calcium carbonate paste remembers the direction of vibration but cannot remember flow direction. We find that colloidal particles in calcium carbonate paste are charged positively in water. On the other hand, as for the paste of magnesium carbonate hydroxide which can remember directions of vibration and flow, colloidal particles are not charged in water. To screen the Coulombic repulsion we added sodium chloride to calcium carbonate paste and confirmed that the paste gets the ability to remember flow direction. Therefore, the ability to remember flow direction strongly depends on the type of the interaction among colloidal particles. Without Coulombic repulsion colloidal particles form clusters via short-ranged interparticle attraction, and the elongation of these clusters along flow direction plays a key role in keeping memory of flow in paste [14].

Recently it has been shown that a memory in paste can be measured, without any information of crack patterns, by checking the anisotropic stress before cracks are formed [15]. This result is expected to be applicable to detect the precursor of how cracks will be formed.

\section{Erasure of memory by ultrasonic waves}

In this section, we report our recent challenge to erase the memory of vibration in paste. First we heated the paste to destroy microstructure related to memory by thermal fluctuation, but it was difficult to control and homogenize the drying gradient under heating, so we just obtained some irregular crack patterns induced by irregular drying. It had been reported that the jamming of granular particles is suppressed by the irradiation of focused ultrasonic waves [16], Therefore, in the next challenge, we irradiated ultrasonic waves to the paste to destroy microstructure by strong acoustic oscillation and erased the memory in paste.

\subsection{Experimental method}

We prepared calcium carbonate paste with a solid volume fraction of $35 \%$, which remembers the direction of initial vibration. We poured the paste into a circular stainless container of $100 \mathrm{~mm}$ diameter and the thickness of the paste is $10 \mathrm{~mm}$ in depth. First we vibrated the paste horizontally for $1 \mathrm{~min}$. at a frequency of 40rpm and the amplitude of $15 \mathrm{~mm}$ by using Triple Shaker NR-80 (Taitec Inc., Tokyo, Japan). Then we lifted up a cubic water tank of $500 \mathrm{~mm}$ in each side until the surface of the water touch the bottom of the circular container from below. At the bottom of the cubic water tank we had placed an ultrasonic wave generator Advantage Multi Sonic Cleaner (Otari Inc., Tokyo, Japan) with a frequency of $37 \mathrm{kHz}, 71 \mathrm{kHz}$ or $102 \mathrm{kHz}$ and with a continuous control of the sound pressure. We irradiated ultrasonic waves to the paste for $1 \mathrm{~min}$., removed the water tank, and dried the paste at room temperature and low humidity. The sound pressure of ultrasonic waves, which is irradiated to the paste, was measured by Hydrophone AQH-100 (Aquasound Inc., Kyoto, Japan). 


\subsection{Effect of ultrasonic waves on crack patterns}

When we just vibrated a paste in one direction for 1 min. and then dried it, we obtain a lamellar crack pattern shown in Fig. 6(a), the direction of which is perpendicular to the direction of the initial vibration. On the other hand, if the paste is vibrated for $1 \mathrm{~min}$., irradiated by ultrasonic waves of $71 \mathrm{kHz}$ and $25 \mathrm{kPa}$ for 1 min, and then dried, an isotropic and cellular crack pattern appears, as is shown in Fig. 6(b). This result indicates that the irradiation of ultrasonic waves erases the memory of vibration in paste and therefore the isotropic and cellular desiccation crack pattern appears.

We systematically studied the effect of applying ultrasonic waves by controlling two parameters, a frequency and a sound pressure of ultrasonic waves. Figure 7 shows that, if the sound pressure exceeds a threshold value indicated as a solid line, the irradiation of ultrasonic waves weakens or erases the memory in paste. It also shows that the value of the threshold is an increasing function of the frequency. The pressure difference between both sides of each particle at the threshold sound pressure is estimated of the order of the yield stress of the paste. The mechanism to erase a memory in paste is now under investigation.

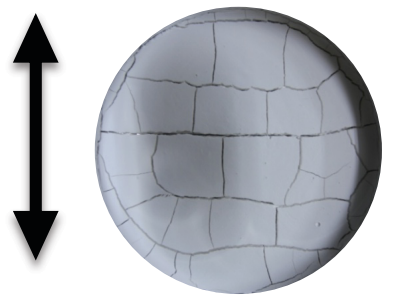

(a)

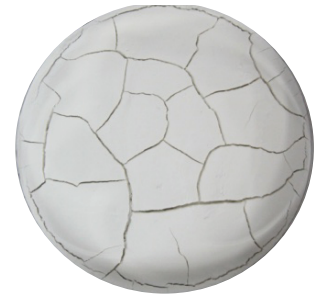

(b)
Fig. 6. Desiccation crack pattern of calcium carbonate paste. The diameter of the circular container is $100 \mathrm{~mm}$ in (a) and (b). (a) The paste is vibrated in one direction for $1 \mathrm{~min}$., then dried. (b) The paste is vibrated for $1 \mathrm{~min}$., irradiated by ultrasonic waves of $71 \mathrm{kHz}$ and $25 \mathrm{kPa}$ for $1 \mathrm{~min}$, and then dried.

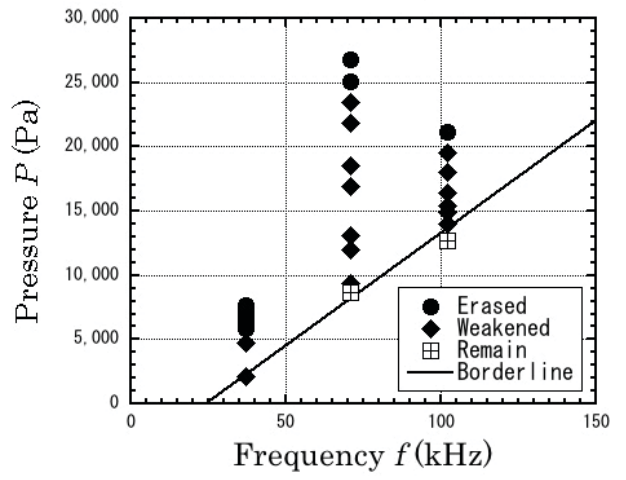

Fig. 7. Effect of the irradiation of ultrasonic waves to paste, as a function of frequency and pressure of applied ultrasonic waves. The paste had been vibrated and had a memory of vibration before it was irradiated by ultrasonic waves. The solid circle denotes isotropic and cellular desiccation crack pattern, the result of which indicates that the memory in paste is erased by the irradiation of ultrasonic waves. The solid rhomboid corresponds to the case where the ultrasonic waves weaken the memory in paste and the open square with plus inside means there is no effect of applying ultrasonic waves.

\section{Conclusions}

We have found that the memory in paste can be visualized as morphology of desiccation crack patterns. This result has two meanings. First, if you observe the crack pattern, you can estimate what happened to the paste before it dries. Second, by imprinting and rewriting the memories in paste, we can control the direction of crack propagation and protect products from serious damages. We have also fond that we can erase the memory in paste by irradiation of ultrasonic waves. Since the memory effect produces easy-breaking directions in paste, the erasure of memory in paste will increase the breaking strength of paste without any easybreaking directions. We consider that this method can be applicable to produce durable construction material by erasing the memory of pouring into molds.

We acknowledge H. Onozaki, Ooshida Takeshi, Sebastien Manneville and Ferenc Kun for valuable discussions. This work was supported by Grant-in-Aid for Scientific Research (KAKENHI) (B)22340112, (C)16K05485 and (C)26400395 of Japan Society for the Promotion of Science (JSPS), by JSPS and HAS under Japan-Hungary Research Cooperative Program and by CST Research Fund of Nihon Univ.

\section{References}

1. L. Goehring, A. Nakahara, T. Dutta, S. Kitsunezaki, S. Tarafdar, Desiccation Cracks and their Patterns: Formation and Modelling in Science and Nature, (Wiley-VCH, Weimheim, Germany, 2015), and references therein.

2. A. Groisman, E. Kaplan, Europhys. Lett., 25, 415 (1994).

3. G. Mueller, J. Volcanol. Geother. Res., 86, 93 (1998).

4. A. Toramaru, T. Matsumoto, J. Geophys. Res., 109, B02205 (2004).

5. L. Goehring, S. W. Morris, Europhys. Lett., 69, 739 (2005).

6. T. Mizuguchi, A. Nishimoto, S. Kitsunezaki, Y. Yamazaki, I. Aoki, Phys. Rev. E, 71, 057122 (2005).

7. A. Nakahara, Y. Matsuo, J. Phys. Soc. J., 74, 1362 (2005) ; J. Stat. Mech.: Theory Exp., P07016 (2005).

8. S. Kitsunezaki, J. Phys. Soc. Jpn. 78, 064801 (2009).

9. H. Nakayama, Y. Matsuo, O. Takeshi, A. Nakahara, Euro Phys. J. E, 36, 1 (2013).

10. M. Otsuki, Phys. Rev. E, 72, 046115 (2005).

11. O. Takeshi, Phys. Rev. E, 77, 061501 (2008); J. Phys. Soc. Jpn., 78, 104801 (2009).

12. S. Kitsunezaki, J. Phys. Soc. Jpn. 79, 124802 (2010) ; Adv. Powd. Tech., 22, 311 (2011).

13. A. Nakahara, Y. Matsuo, Phys. Rev. E, 74, 045102(R) (2006).

14. Y. Matsuo, A. Nakahara, J. Phys. Soc. Jpn., 81, 024801 (2012).

15. S. Kitsunezaki, A. Nakahara, Y. Matsuo, Europhys. Lett., 114, 64001 (2016).

16. P. Lidon, N. Taberlet, S. Manneville, Softmatter, 12, 2315 (2016). 